\title{
THE ACTION OF CHOLINERGIC AGONISTS ON THE SQUID STELLATE GANGLION GIANT SYNAPSE ${ }^{1}$
}

\author{
E. F. STANLEY \\ Marine Biological Laboratory, Woods Hole, Massachusetts 02543 and Department of Neurology, Johns Hopkins School of \\ Medicine, Baltimore, Maryland 21205
}

Received December 27, 1983; Revised February 28, 1984; Accepted February 28, 1984

\begin{abstract}
Although the giant synapse in the squid stellate ganglion has served as a model in the understanding of the ionic and electrical changes that occur during the release of transmitter from nerve terminals, little is known about the pharmacology of this synapse or the identity of its neurotransmitter. In the present study, the suggestion that acetylcholine (ACh) is the excitatory transmitter at this synapse was tested by exploring the actions of cholinergic agents on the pre- and postsynaptic giant axons and on the excitatory postsynaptic potential (EPSP). A novel arterial perfusion technique that circumvents the diffusion barrier from the bathing medium to the synapse has been used to demonstrate a depolarizing action of $\mathrm{ACh}$ and its agonist carbachol on the post- but not the presynaptic axon. The cholinergic receplors are distinct from receptors activated by amino acids, such as glutamate, have a reversal potential of about $-48 \mathrm{mV}$, are anion-permeable, and desensitize without blocking the EPSP. It is concluded that these receptors are involved in an inhibitory input onto the postsynaptic giant axon and, therefore, that $\mathrm{ACh}$ is most probably not the transmitter at the squid giant synapse.
\end{abstract}

The giant synapse in the squid stellate ganglion has served as a model in the study of the ionic permeability changes and electrical events that are associated with the release of transmitter from the nerve terminal (e.g., Bullock and Hagiwara, 1957; Takeuchi and Takeuchi, 1962; Katz and Miledi, 1967; Kusano et al., 1967; Miledi, 1973; Llinas and Nicholson, 1975; Llinas et al., 1981; Charlton et al., 1982). The large size of the presynaptic axon terminal (Young, 1939), allowing direct microelectrode recording (Bullock, 1948) and current passing, is the principal reason for the usefulness of this preparation. In contrast, this preparation may be one of the least well understood preparations in terms of its pharmacology; in particular, the transmitter active at this synapse has not been identified, and little is known about the receptors on the postsynaptic axon. The main reason the pharmacological properties of this neurobiologically important synapse have not been explored is the substantial diffusion barrier from the bathing medium to the syn-

\footnotetext{
${ }^{1} \mathrm{I}$ am indebted to a number of individuals in the course of carrying out and writing this study, including E. Aizenman and Drs. W. J. Adelman, Jr. (National Institutes of Health Biophysics Laboratory, Marine Biological Laboratory), M. P. Charlton (University of Toronto), S. J. Smith (Yale University), G. J. Augustine, Jr. (University of California, Los Angeles), and P. Talalay. I thank Ms. C. Barlow-Salemi for secretarial assistance. This work was supported by Grant R03 MH38359-01 from the National Institute of Mental Health.
}

apse, noted by several workers (Bryant, 1958; Webb et al., 1966; Kelly and Gage, 1969; Lester, 1970; Stanley and Adelman, 1982).

Previous studies have proposed two substances as excitatory transmitters at this synapse: glutamate (GLU) (Miledi, 1967) and acetylcholine (ACh) (Webb et al., 1966). These proposals are consistent with morphological studies which have shown that the vesicles in the presynaptic axon terminal are large and clear (Froesch and Martin, 1972; Pumplin and Reese, 1978). The evidence supporting GLU includes blockade (desensitization ?) of the excitatory postsynaptic potential (EPSP) with bath application (Kelly and Gage, 1969) and depolarization of the postsynaptic axon membrane with iontophoresis (Miledi, 1967, 1969, 1972), although the difficulty of iontophoresis of agents into this preparation was noted (see "Discussion"). However, the hypothesis that GLU is the transmitter has been questioned due to the observation that the depolarization evoked by GLU has a different reversal poential $\left(E_{R}\right)$ (Miledi, 1969) from that of the EPSP (Miledi, 1969; Llinas et al., 1974).

The evidence in favor of cholinergic transmission is based on the presence of cholinesterase in the stellate ganglion (Nachmansohn and Meyerhof, 1941; Bryant and Brzin, 1966) and its localization near the giant synapse in morphological studies (Brzin et al., 1975), and the demonstration of $\mathrm{ACh}$ and choline acetyltransferase 
(CAT) in segments of the ganglion containing the giant synapse (Rosenberg et al., 1966; Webb et al., 1966). However, cholinergic markers have also been reported in non-neural cells (Heumann et al., 1981). Studies attempting to demonstrate a physiological action of ACh at the giant synapse have resulted in, at best, inconclusive results. Application of $\mathrm{ACh}$ in the bathing medium failed to depolarize the postsynaptic axon, and synaptic transmission was blocked only after long periods of treatment (16 to $90 \mathrm{~min}$ ) in very high concentrations of $\mathrm{ACh}$ $\left(5 \times 10^{-2}\right.$ м) (Bryant, 1958; Webb et al., 1966). The failure of ACh to cause a physiologically detectable action on the postsynaptic giant axon was attributed (correctly, see below) to the diffusion barrier (Webb et al., 1966).

In view of the evidence questioning GLU as the excitatory transmitter at this synapse, the present study was undertaken to re-evaluate the possible role of $\mathrm{ACh}$, since all of the characteristic cholinergic markers have been identified in the stellate ganglion. In order to study the action of ACh at this synapse however, it is first necessary to solve the technical problem enountered in previous studies, of the diffusion barrier from the bathing medium to the giant synapse itself. We have recently described a novel arterial perfusion technique that circumvents this barrier and allows the virtually immediate introduction of agents into the stellate ganglion (E. F. Stanley and W. J. Adelman, Jr., 1982, and submitted for publication). The hypothesis that ACh may be the transmitter at this synapse was examined by testing the effect of cholinergic agonists on this axon. It is shown that there are indeed cholinergic receptors on the postsynaptic site, but that these receptors have characteristics that are not consistent with ACh acting as an excitatory transmitter at the squid stellate ganglion giant synapse.

\section{Materials and Methods}

Experiments were carried out on small squid of mantle length 100 to $140 \mathrm{~mm}$. The dissection, mounting, and perfusion technique have been described (E. F. Stanley and W. J. Adelman, Jr., submitted for publication) but are summarized below.

The squid was decapitated, the mantle was cut along the ventral midline, and the internal organs, excepting the gut, were removed. The muscle wall of the gut was cut up either side, and the gut was carefully removed, taking care not to damage the underlying blood vessels or the nerves from the cercbral ganglia. The aorta was cannulated caudally, close to the point where a single branch, the common stellate artery, penetrates the gut wall and bifurcates to supply the two ganglia (the right and left stellate arteries). A ligature was tied on the rostral side of the common stellate artery. 'Ihe left stellate artery was tied off, and the right stellate ganglion was removed, together with $10 \mathrm{~mm}$ of the prenerve containing the second-order giant axon from the cerebral ganglion, and $15 \mathrm{~mm}$ of the medial stellar nerve, which contains the last, and largest, of the third-order giant axons. The preparation was transferred to a chamber consisting of a silastic-coated Petri dish and placed on the stage of a dissecting microscope. A window was cut in the smooth muscle capsule of the ganglion to allow access of microelectrodes to the pre- and postsynaptic giant axons. Perfusion was achieved by forcing artificial sea water (ASW, composition in millimolar concentration: $\mathrm{NaCl}, 423.0 ; \mathrm{KCl}, 9.0 ; \mathrm{CaCl}_{2}, 9.3 ; \mathrm{MgCl}_{2}, 25.5$; $\mathrm{MgSO}_{4}, 25.5$; HEPES, 5.0; $\mathrm{pH} 7.2$ ) through the ganglion under pressure from an $\mathrm{O}_{2}$ gas tank. The preparation was well maintained by this method; transmission continued for several hours of study without further oxygenation.

Test solutions were introduced into the ganglion by manually turning the tap on a micro-stopcock (Hamilton). The test substances were housed in containers under the same $\mathrm{O}_{2}$ pressure. With this "constant pressure" technique, pressure changes during switching from one solution to another were avoided so that microelectrode recording could be used to evaluate the effects of the test solutions without any movement artefact. Flow rates ranged from 0.1 to $0.5 \mathrm{ml} / \mathrm{min}$, depending in part on leaks through small artery branches that were not ligatured. Experiments were carried out at room temperature $\left(22^{\circ} \mathrm{C}\right)$.

Stimulation and recording techniques. The prenerve was stimulated by a square wave pulse of electricity $(0.1$ msec) originating from a stimulator (Grass S48) via a stimulus isolation unit and a pair of silver wire electrodes hooked under the nerve. Intracellular recording was carried out by conventional microelectrode techniques. A 3 $\mathrm{M}$ potassium chloride-filled micropipette (5 to 15 megohms) was connected by a holder and probe to a WPI S7000 microelectrode amplifier. Currents were passed intracellularly by means of a second microelectrode $(<10$ megohms) filled with $3 \mathrm{~m}$ potassium acetate connected to a constant current output from the $\mathrm{S} 7000$. The ground electrode was a heavily chlorided coil of silver wire. The output of the amplifier was displayed on a storage oscilloscope (Tektronix D11) to record action potentials and synaptic potentials, while resting potentials were recorded on the digital meter of the $\mathbf{S} 7000$.

\section{Results}

Effect of acetylcholine and carbachol (CARB). Infusion of ACh (1 mM) resulted in an abrupt depolarization of the postsynaptic giant axon followed by a gradual repolarization toward the resting level (Fig. 1). Switching the perfusion solution from ACh to CARB (1 mM) did not affect the resting membrane potential. After washout with plain ASW, however, CARB also depolarized the postsynaptic membrane. The amplitude of the depolarization was related to the concentration of CARB, and depolarizations were detected at concentrations as low as $0.1 \mathrm{mM}$. The possibility that this was an indirect action caused by inducing the release of a transmitter from a nerve terminal synapsing on the giant axon was ruled out by blocking synaptic transmission with $\mathrm{Cd}$ (1 mM) (E. F. Stanley and W. J. Adelman, Jr., submitted for publication). CARB-induced depolarizations were still observed. Thus, both $\mathrm{ACh}$ and its agonist CARB depolarize the postsynaptic giant axon and this action desensitizes with prolonged application. 'Ihese results suggest the presence of a receptor on the postsynaptic membrane that is activated by cholinergic agents. Fur- 


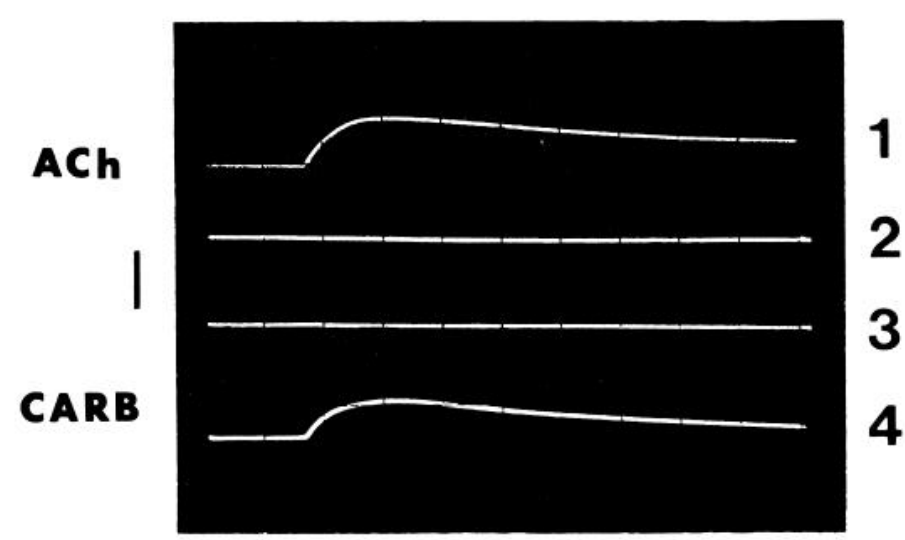

$\Lambda$

Figure 1. Effect of infused ACh or CARB on the postsynaptic giant axon. Each trace is the membrane potential recorded intracellularly during the change of perfusion solution initiated at the arrowhead and continued to the end of the trace. Trace 1, Perfusion solution changed from sea water to ACh. Trace 2, From ACh to $1 \mathrm{~mm}$ CARB. Trace 3, From CARB to ACh. Trace 4 , From sea water to CARB. The resting membrane potential was $64 \mathrm{mV}$ at the beginning of the experiment and $64.5 \mathrm{mV}$ at the end. Note that $\mathrm{ACh}$ and $\mathrm{CARB}$ evoke a depolarization in the postsynaptic giant axon that repolarizes spontaneously and desensitizes to the other agent. The delay between changing the test solution and the onset of the depolarizations recorded in traces 1 and 4 is due to the time taken to clear the sea water from the dead space in the cannula and artery. Calibration: vertical, $10 \mathrm{mV}$; horizontal, $10 \mathrm{sec}$.

ther studies were carried out with CARB, rather than $\mathrm{ACh}$, because of metabolic stability.

Effect of CARB on the EPSP. When the prenerve was stimulated, the resulting postsynaptic EPSP usually reached threshold so that an EPSP/action potential complex was recorded. In the occasional ganglion, however, the EPSP evoked at low stimulus intensities failed to reach threshold, and the effect of cholinergic agents on the EPSP could be evaluated.

Infusion of $1 \mathrm{mM}$ CARB during the stimulation of the prenerve resulted in a decline in the EPSP that had a similar time course to the depolarization of the postsynaptic membrane. During the continued infusion of $\mathrm{CARB}$, the membrane potential recovered toward preinfusion values, as did the EPSP (Fig. $2 a$ ). This result was in contrast to the effect of GLU, which is known to depolarize the postsynaptic giant axon (Miledi, 1967) and to block (desensitize?) the EPSP (Kelly and Gage, 1969). These findings were confirmed using the infusion technique (Fig. 3, trace 1). Thus, with GLU, the postsynaptic giant axon depolarizes and the EPSP declines during desensitization. Since the EPSP recovers during the continued infusion of $\mathrm{CARB}$, this compound reduces the EPSP without blocking the receptors activated by the endogenous transmitter. In addition, it was demonstrated that CARB can activate a set of receptors that is independent of those activated by glutamate, since even after prolonged desensitization to GLU, a CARB response could still be detected and vice versa (Fig. 3 ).

Effect of CARB on the presynaptic axon terminal. The observation that CARB reduced the EPSP independ- ently of the endogenous transmitter receptors suggests either that it acts on the presynaptic nerve terminal, resulting in the inhibition of transmitter release, or it reduces the $R_{\mathrm{IN}}$ of the postsynaptic giant axon.

\section{a.}

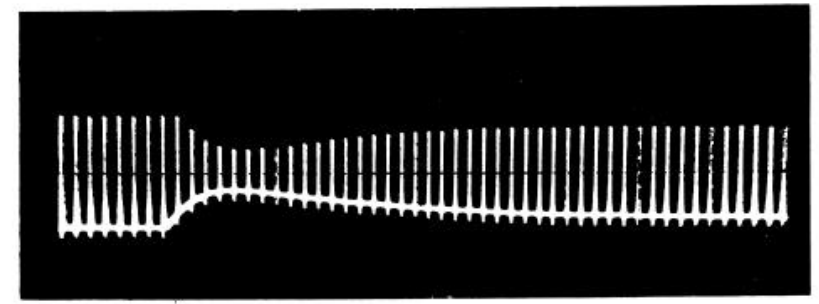

b.

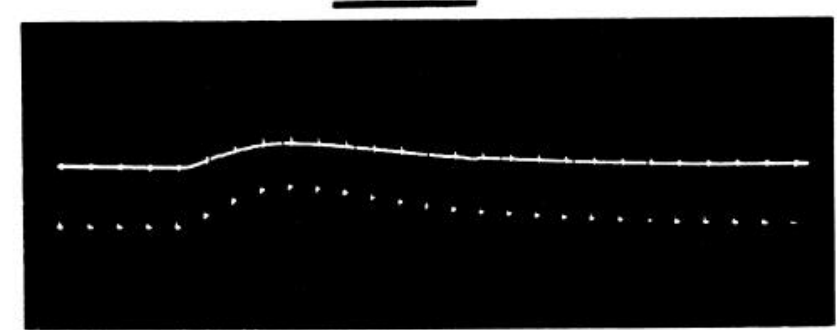

$\boldsymbol{\Lambda}$

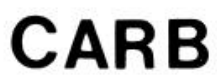

Figure 2. $a$, Effect of CARB on the EPSP. EPSP values were recorded during the infusion of CARB (1 $\mathrm{mm})$ by stimulating the prenerve at a frequency of $1 \mathrm{~Hz}$. Note the decline in the EPSP during the CARB-induced depolarization but its subsequent gradual recovery. Resting membrane potential was 64.5 mV. $b$, Effect of CARB on $R_{\mathrm{IN}}$. A 40-nA, 200-msec hyperpolarizing current pulse was passed into the giant axon during the infusion of CARB. Note the reduction in the current-induced hyperpolarization during the CARB response. Resting membrane potential was $63.0 \mathrm{mV}$. Calibration: $10 \mathrm{mV}, 10 \mathrm{sec}$.

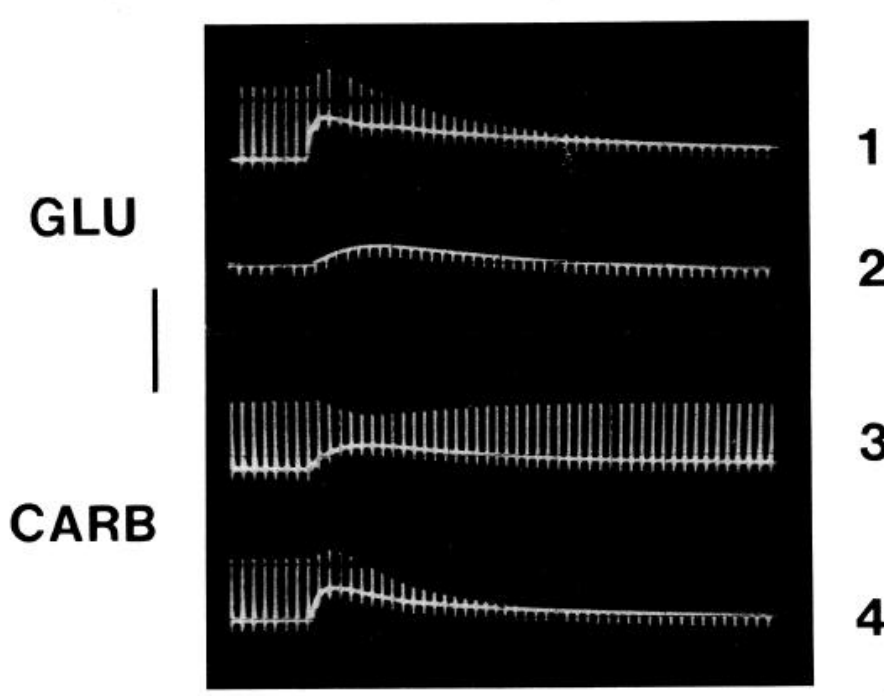

$\Lambda$

Figure 3. Comparison of the actions of GLU and CARB on the membrane potential and EPSP (see Fig. 2a). Perfusion solutions were changed at the arrowhead. Trace 1, Perfusion solution changed from sea water to GLU. Trace 2, From GLU to ACh. Trace 3, From sea water to ACh. Trace 4, From ACh to GLU. Note the blockade of the EPSP by GLU but not CARB, and the depolarizations evoked by either substance after desensitization to the other. Calibration: $10 \mathrm{mV}, 10 \mathrm{sec}$. 


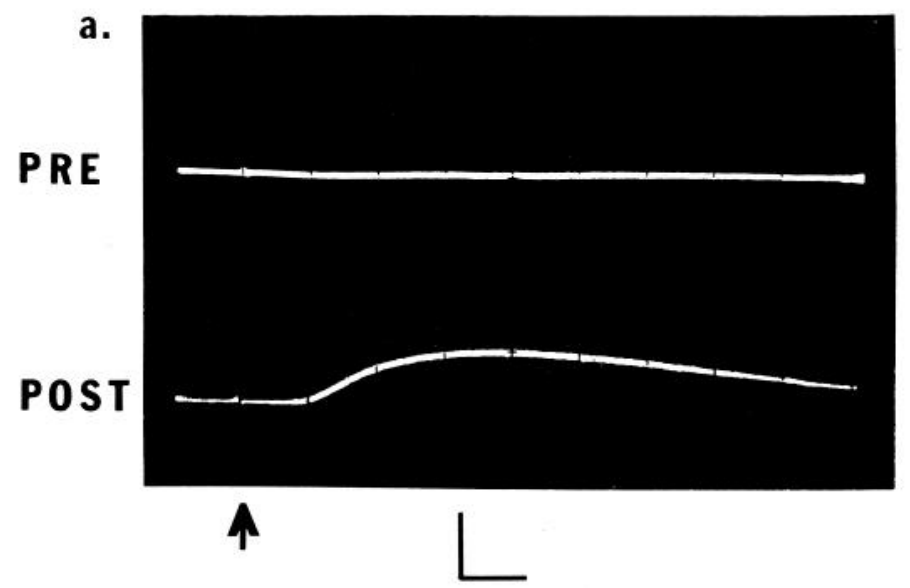

b.

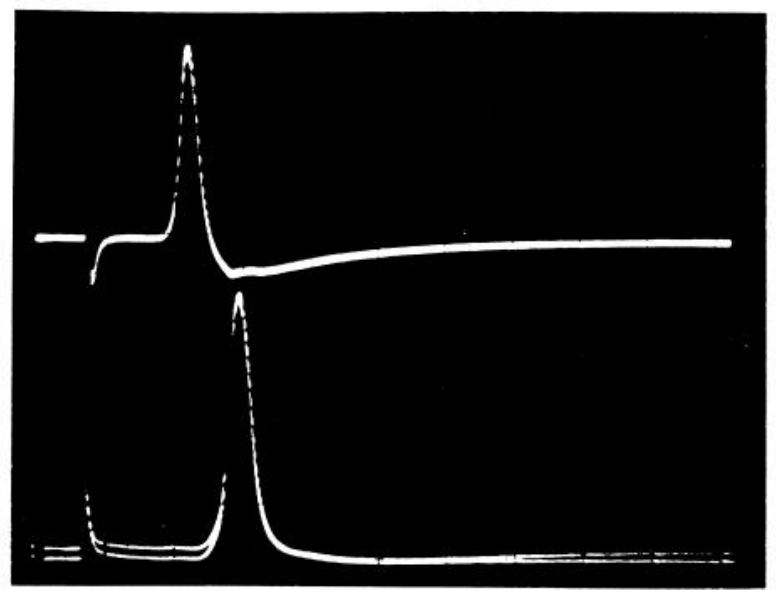

$\mathbf{S}$

Figure 4. Comparison of the effect of CARB on the pre- and postsynaptic giant axons. $a$, Membrane potentials recorded simultaneously from the pre- $(P R E)$ and postsynaptic (POST) giant axons during the infusion of CARB onset at the arrow. Resting membrane potentials were PRE $65.5 \mathrm{mV}$ and POST $64 \mathrm{mV}$. Note the depolarization of the postsynaptic giant axon but lack of effect on the presynaptic axon. Calibration: $5 \mathrm{mV}, 5$ sec. $b$, Action potentials recorded simultaneously from the pre(upper) and postsynaptic (lower) giant axons during the infusion of CARB (at a lower amplification than a). Each trace is the superimposed sweeps of two records, one prior to CARB and the other at approximately the peak of the postsynaptic CARB depolarization. Note the lack of effect of CARB on the presynaptic membrane potential or action potential at a time when a depolarization of the postsynaptic axon is clearly evident. Resting membrane potentials were as above. $S$, stimulus artifact. Calibration: $20 \mathrm{mV}$ and $2.0 \mathrm{msec}$.

The effect of CARB on the nerve terminal was evaluated by intracellular recording from the presynaptic giant axon. No changes were detected in the presynaptic axon resting membrane potential or action potential (Fig. 4). Although these observations do not rule out a reduction in transmitter release by CARB, they suggest that the effect of this agent on the EPSP is a consequence of its actions on the postsynaptic membrane, perhaps by a reduction in the input resistance $\left(R_{\mathrm{IN}}\right)$.

Effect of CARB on the postsynaptic giant axon input resistance. The effect of CARB on the $R_{\mathrm{IN}}$ was evaluated by passing a $200-\mathrm{msec}$ hyperpolarizing pulse into the postsynaptic axon via a second microelectrode during the infusion of $1 \mathrm{mM}$ CARB. The current/voltage relation over the range of the CARB-induced depolarization (see Fig. $5 c$ ) approximates to a straight line, and, thus, any decline in the $R_{\mathrm{IN}}$ can be attributed to the action of the cholinergic agent. The membrane potential depolarized by $3.5 \mathrm{mV}$, and the $R_{\mathrm{IN}}$ declined from $2.0 \times 10^{5} \mathrm{ohms}$ to $1.4 \times 10^{5}$ ohms at the peak of the response (Fig. $2 b$ ). This result suggests that the action of CARB involves a large increase in the ion permeability of the postsynaptic giant axon.

Reversal potential of the CARB depolarization. A clue to the nature of the permeability change may be obtained by determining the reversal potential of the CARBinduced depolarization $\left(E_{\mathrm{CARB}}\right)$. This was determined by passing hyper- or depolarizing current pulses into the postsynaptic giant axon by means of a second micropipette during the infusion of CARB. Since the $E_{\mathrm{CARB}}$ was close to the resting potential, and the I/V curve was approximately linear over the membrane potential range studied, the $E_{\mathrm{CARB}}$ could be determined by extrapolation

a.
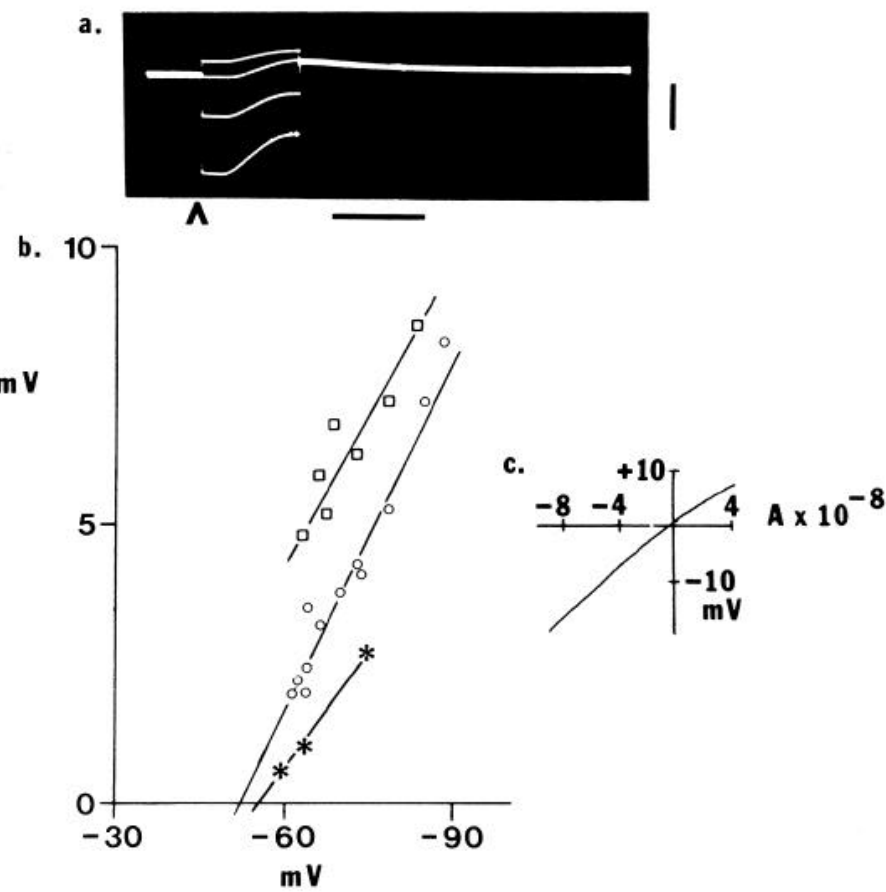

Figure 5. Estimation of the $E_{\mathrm{CARB}} \cdot a$, Intracellularly recorded membrane potentials from the postsynaptic giant axon during the infusion of CARB (onset at the arrowhead) and the simultaneous passing of a 10 -sec current pulse via a second microelectrode $<200 \mu \mathrm{m}$ away. Current pulses were from top to bottom: $+15 \mathrm{nA}, 0 \mathrm{nA}$ (resting membrane potential), $-30 \mathrm{nA}$, and $-60 \mathrm{nA}$. The CARB-induced depolarization increases markedly at the more negative membrane potentials. These data are a part of line $O$ in b. Calibration: $10 \mathrm{mV}, 10$ sec. $b$, Plot of amplitude of CARB-induced depolarization ( $y$ axis) against membrane potential for CARB dissolved in filtered sea water (O) or ASW (*). The relation of membrane potential to GLUinduced depolarization over a similar range is given for comparison ( $\square$ ). Resting membrane potentials in all three experiments were 63 to $64 \mathrm{mV}$. Straight lines have been fitted and extrapolated to the $x$ axis to estimate the $E_{\mathrm{CARB}}$ values. $c$, Current/voltage relation for the postsynaptic axon denoted by $\mathrm{O}$ in $b$. 
without resorting to the use of blocking agents to eliminate voltage-sensitive ion currents.

The amplitude of the CARB-induced depolarizations was plotted against the membrane potential set by the current pulse. Alternatively, the initial rate of change of the CARB depolarization, measured from Polaroid photographs, was plotted. Both measurements gave very close $E_{\mathrm{R}}$ estimates.

The CARB-induced response was found to decrease steeply with membrane potential depolarization (Fig. $5 a$ ) and was calculated to reverse (by extrapolation of the regression line fitted by the least squares method) at -45 \pm (SE) $4 \mathrm{mV}$ (three experiments) in filtered sea water, and $-48 \pm 4 \mathrm{mV}(n=4)$ in ASW (e.g., Fig. $5 b)$. These values are not significantly different. The relationship between membrane potential and the amplitude of a depolarization induced by GLU was examined over a similar voltage range to compare with the $E_{\mathrm{CARB}}$. Clearly, the amplitude of the GLU-induced response was less dependent on the membrane potential, suggesting a more positive $E_{\mathrm{GLU}}$, consistent with previous findings (Miledi, 1969).

Effect of $\mathrm{Cl}^{-}$replacement on the CARB response. The similarity of the $E_{\mathrm{CARB}}$ to the calculated $E_{\mathrm{Cl}}$ (see "Discussion") suggested that the cholinergic receptor may be an anion-selective channel. The role of chloride in the CARB depolarization was evaluated by replacing $50 \%$ of the external chloride with methylsulfonate $\left(\mathrm{MSO}^{-}\right)$(total $\mathrm{Cl}^{-} 286 \mathrm{~mm}, \mathrm{MSO}^{-} 211 \mathrm{mE}$ ). This resulted in an

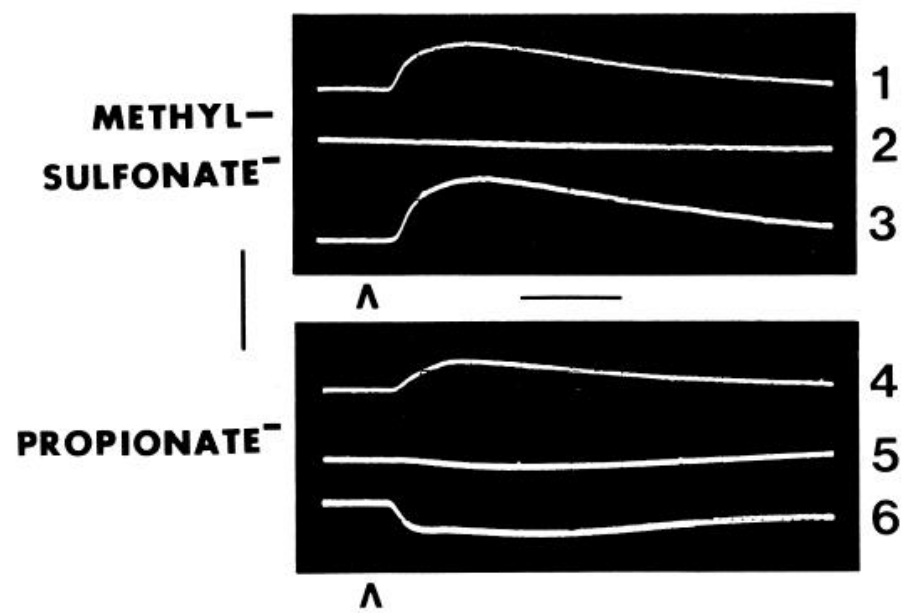

Figure 6. Effect of external $\mathrm{Cl}^{-}$replacement on the CARBinduced membrane potential change. Perfusion solutions were changed at the arrowhead. Trace 1, (resting membrane potential, $-60 \mathrm{mV})$ : Solution was changed from ASW to CARB; note the $4.6-\mathrm{mV}$ depolarization. Trace 2, from ASW to low $\mathrm{Cl}^{-}$ (replacement of approximately $50 \%$ external $\mathrm{Cl}^{-}$with methylsulfonate): There is little effect on the membrane potential. Trace 3, from low $\mathrm{Cl}^{-}$to low $\mathrm{Cl}^{-}$plus CARB: Note increased 6.3-mV depolarization. Trace 4, (new experiment, resting potential, $-65.5 \mathrm{mV}$ ), from ASW to CARB: Note the $3-\mathrm{mV}$ depolarization. Trace 5, from ASW to low $\mathrm{Cl}^{-}$(replacement with propionate): There is a small hyperpolarization $(<1 \mathrm{mV})$, followed by a small depolarization (total $2 \mathrm{mV}$ from the ASW resting potential). Trace 6 , from low $\mathrm{Cl}^{-}$to low $\mathrm{Cl}^{-}$plus CARB: CARB induced a hyperpolarization of $3.7 \mathrm{mV}$. Note also a possible second component to the response, possibly due to a superimposed $\mathrm{Cl}^{-}$flux.

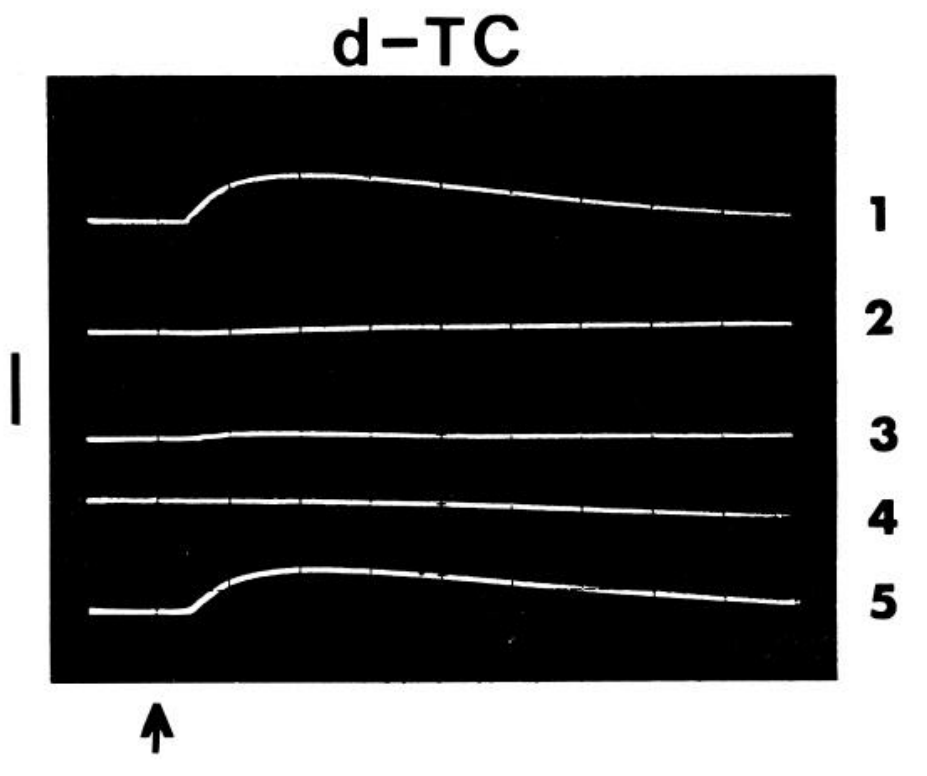

Figure 7. Effect of $d$-TC on the CARB-induced membrane potential depolarization. Trace 1, Change of perfusion solution from ASW to CARB (1 mM); CARB induces a 7.5-mV depolarization. Trace 2, ASW to $d$-TC (1 mM). Trace 3, $d$-TC to $d$-TC plus CARB; a very small depolarization $(<1 \mathrm{mV})$ is recorded in response to CARB. Trace 4, $d$-TC to ASW. Trace 5, ASW to $\mathrm{CARB}$, demonstrating the recovery of the response on washout of $d$-TC. Calibration: $5 \mathrm{mV}, 10 \mathrm{sec}$.

increase in CARB-induced depolarizations (Fig. 6, top). Thus, the CARB response appeared to be dependent on the external anion concentration.

An equimolar substitution of external $\mathrm{Cl}^{-}$was also carried out with sodium propionate $\left(\mathrm{PRO}^{-}\right)$. In $\mathrm{PRO}^{-}$ ASW, CARB treatment resulted in a hyperpolarization of the postsynaptic giant axon (Fig. $5 b$ ). It was also noted that after repeated trials of CARB in the PRO ${ }^{-}$ASW and the washout with normal $\mathrm{Cl}^{-} \mathrm{ASW}$, the CARB depolarization increased in amplitude, from $3.5 \mathrm{mV}$ to $7.2 \mathrm{mV}$.

Effect of d-tubocurarine (d-TC). $d$-TC infused into the ganglion blocked the action of CARB but only at high concentrations (1 mM) (Fig. 7).

\section{Discussion}

The principal findings of this study are: first, the demonstration of a direct physiological action of $\mathrm{ACh}$ on the postsynaptic giant axon in the squid stellate ganglion; second, evidence suggesting an anion channel for the cholinergic receptor; and third, the failure of $\mathrm{ACh}$ to block synaptic transmission directly.

The merits of the arterial infusion technique for the access of substances, primarily ions, into the giant synapse have been discussed (E. F. Stanley and W. J. Adelman, Jr., submitted for publication). The present study employs arterial infusion as a method of evaluating the effects of pharmacological agents, as an alternative to the more usual techniques of iontophoresis and bath application. In other experimental preparations, iontophoresis is usually the preferred technique, because of 
the fast access of drugs to the cell membrane and the reasonably precise control over the region of the preparation that is exposed to the drug. This technique is difficult in the stellate ganglion-giant synapse preparation, however (Miledi, 1969, 1972), and only GLU has been reported to have any effect on membrane potentials in this synapse (Miledi 1967, 1969, 1972). Bath application has been even less successful at the giant synapse. Agents that are known to act quickly on different aspects of synaptic transmission in other preparations take many minutes or even hours at the giant synapse due, presumably, to the overlying diffusion barrier (Bryant, 1958; Webb et al., 1966; Kelly and Gage, 1969; Lester, 1970; E. F. Stanley, submitted for publication).

Aortic perfusion is intermediate between the above two approaches. Unlike bath application, the test agent reaches the cell under study fast enough to evoke a biological response, comparing quite favorably (in terms of time of onset to peak) with reports of iontophoresis of $\mathrm{ACh}$ onto other molluscan neurons (Chiarandini et al., 1967; Blankenship et al., 1971; Kehoe 1972a). Thus, arterial infusion results in a detectable depolarization of the postsynaptic giant axon with ACh or GLU, whereas bath application of ACh (Bryant, 1958; Webb et al., 1966) or GLU (Kelly and Gage, 1969) does not. Unlike iontophoresis, the technique is reliable; similar $\mathrm{ACh}$ responses were observed in all ganglia tested (over 25), and, furthermore, dose-response relations may be examined since the concentration of the test agent in the treatment solution can be accurately controlled.

The main drawback to arterial perfusion is that, as with bath application, the whole preparation undergoes the same drug treatment. Therefore, it is necessary to ensure that the observed responses are due to the test agent and are not secondary to some other action and that the recorded effects occur close to the recording electrode. The observation that similar CARB responses were still observed in the presence of $\mathrm{Cd}^{2+}$ rules out the possibility that CARB induces the release of some other neurotransmitter substance by normal $\mathrm{Ca}^{2+}$-related mechanisms. However, the possibility that the test substance may act at a site on the postsynaptic giant axon that is not precisely at the recording electrode is more difficult to rule out. The best evidence in support of the localization of $\mathrm{ACh}$ receptors close to the recording site is the abrupt onset of depolarizations induced by the cholinergic or glutaminergic agents, particularly when the method of introduction of agents is taken into consideration. Some mixing, and hence dilution, of the substance with ASW must occur in the cannula and artery, prior to its access to the giant synapse. Furthermore, ACh in concentrations up to $10 \mathrm{~mm}$ does not depolarize the giant axon itself (Yeh and Narahashi, 1974), and in other studies only slow, small amplitude depolarizations were recorded from the neurons in the giant fiber lobe, proximal to the giant synapse-consistent with an action distant from the cell bodies and close to the giant synapse. This is in contrast to large responses, usually hyperpolarizations, recorded from neurons in the spaces between the stellar nerves (E. F. Stanley, personal observations).

The access of substances to the synapse did not appear to be significantly impeded by a blood-nerve barrier. Such a barrier has been reported for the central ganglia of the cuttlefish Sepia officinalis, in which the access of proteins (Abbott et al., 1981) and uncharged nonelectrolytes of molecular radius greater than $5 \AA$ is restricted (Abbott et al., 1982). The observations that kainate (molecular weight 213) and other amino acids (Stanley, 1983), carbachol (183), and even $d$-TC (682) have pharmacological actions on the giant synapse suggest that such a barrier is not of great importance in the stellate ganglion. Further studies will be necessary to evaluate fully the permeability of the blood vessels in this preparation.

The existence of cholinergic receptors on the postsynaptic giant axon is supported by the following observations: $(a)$ a concentration-dependent depolarization by cholinergic agents; $(b)$ the desensitization of the response to both the continued administration of $\mathrm{ACh}$ or its agonist CARB; (c) the independence of this response from the depolarization induced by another agent that has biological action at this synapse, GLU; and $(d)$ the blockade of the depolarization by $d$-TC (albeit at high concentrations).

The CARB-induced depolarization was found to be associated with a large decrease in the $R_{\mathrm{IN}}$. This finding indicates that the action of CARB results in a large increase in membrane permeability, i.e., the opening of ion channels. At first sight this is consistent with the action of $\mathrm{ACh}$ as an excitatory transmitter at the giant synapse. Therefore, further studies were undertaken to examine whether these cholinergic receptors were similar to those activated by the endogenous transmitter.

As noted in the introduction, the $E_{\mathrm{R}}$ for the endogenous transmitter is in the positive membrane potential range ( +14 to $+40 \mathrm{mV})$. The $E_{\mathrm{CARB}}$ was found to be much closer to the resting membrane potential, with a value of about $-50 \mathrm{mV}$. This suggests either a cation channel permeable primarily to $\mathrm{K}^{+}$, but with a small permeability to $\mathrm{Na}^{+}$(permeability only to $\mathrm{K}^{+}$ions would result in a CARB-induced hyperpolarization), or an anion channel, similar to cholinergic receptors in other molluscan preparations (see below).

Since $\mathrm{Cl}^{-}$is actively pumped across the membrane (Keynes, 1963; Russell, 1976), the $E_{\mathrm{Cl}}$ is more positive than the resting potential. Therefore, opening of ion channels permeable to $\mathrm{Cl}^{-}$would be expected to result in a membrane depolarization, consistent with the action of CARB. The theoretical $E_{\mathrm{Cl}}$ may be calculated from the concentration ratios of ions across the membrane, as the activities of $\mathrm{Cl}^{-}$are approximately equal in sea water or axoplasm (Keynes, 1963). Based on the known external $\mathrm{Cl}^{-}$concentrations and an internal $\mathrm{Cl}^{-}$of $108 \mathrm{mM}$ (Keynes, 1963), the $E_{\mathrm{Cl}}$ values are $-39.4 \mathrm{mV}$ in sea water and $-38.4 \mathrm{mV}$ in ASW as compared with experimentally determined $E_{\mathrm{CARB}}$ values of $-45 \mathrm{mV}$ and $-48 \mathrm{mV}$, respectively. Thus, the $E_{\mathrm{CARB}}$ is close to, but not the same as, the theoretical $E_{\mathrm{Cl}^{-}}$. Note that an error in the determination of the $E_{\mathrm{CARB}}$ if the ACh receptors were located at a distance from the recording/current passing electrodes would be more likely to result in an $E_{\mathrm{CARB}}$ estimate that is too positive rather than too negative, since more current would have to be passed to approach the $E_{\mathrm{R}}$ (see 
Ginsborg, 1967). Thus, the true $E_{\mathrm{CARB}}$ must lie within the range of the experimentally determined value and the resting potential of $-65 \mathrm{mV}$.

Further evidence in support of an anion-permeable channel was obtained by replacing a large fraction of the external $\mathrm{Cl}^{-}$with other anions. $\mathrm{MPO}^{-}$substitution resulted in an increase in the CARB-induced depolarization, as would be predicted by a shift in the $E_{\mathrm{Cl}}$ toward more positive values. Replacing $\mathrm{Cl}^{-}$with $\mathrm{PRO}^{-}$resulted in a CARB-induced hyperpolarization of the postsynaptic axon membrane. When the $\mathrm{PRO}^{-}$was washed out with normal $\mathrm{Cl}^{-} \mathrm{ASW}$, the CARB-induced depolarization was found to be augmented. These results can be explained simply if we presume that the receptor channel is permeable to the $\mathrm{PRO}^{-}$ion. Since this ion is absent from the axoplasm, its concentration gradient across the membrane is exceedingly high, giving a very low (but unknown) $E_{\mathrm{Pro}}$. Thus, opening of the cholinergic receptor channels due to the action of CARB will result in a shift in the membrane potential toward the $E_{\mathrm{PRO}}$, and, if this ion current is larger than the $\mathrm{Cl}^{-}$current, an hyperpolarizing response could well occur. If sufficient $\mathrm{PRO}^{-}$ entered the axon as a result of repeat trials with CARB, when the preparation is returned to ASW and CARB is again applied, $\mathrm{PRO}^{-}$ions in the axoplasm would diffuse out of the axon and increase the CARB-induced membrane depolarization.

If the cholinergic receptor ionophore is selectively permeable to anions, the question remains why the experimentally determined $E_{\mathrm{CARB}}$ does not correspond more closely to the $E_{\mathrm{R}}$ of the principal anion, $\mathrm{Cl}^{-}$. Several possible explanations exist. First, the receptor may be permeable to $\mathrm{SO}_{4}{ }^{2-}$, the only other anion in the ASW. Although this might be unusual as compared to other molluscan cholinergic receptors (see Kehoe, 1972b), it might not be entirely unexpected in view of the unusually high permeability to $\mathrm{PRO}^{-}$. Second, the receptor could be partially permeable to a cation such as $\mathrm{Na}^{+}$or $\mathrm{Ca}^{2+}$; or, third, the estimates for $\mathrm{Cl}^{-}$in the giant axon itself may not be correct for the synaptic region. The latter is a possibility since a large efflux of $\mathrm{Cl}^{-}$due to activation of the cholinergic receptor would probably quickly overload the $\mathrm{Cl}^{-}$pump, which accumulates $\mathrm{Cl}^{-}$rather slowly at a rate of $12 \mathrm{pmol} \times \mathrm{cm}^{2}$ (Russell, 1976). Thus, if we assume that $\mathrm{Cl}^{-}$is the principal permeant ion, the internal $\mathrm{Cl}^{-}$concentration, with a $48-\mathrm{mV}$ reversal potential in ASW, would be $73.8 \mathrm{~mm}$.

Cholinergic anion channels with a permeability to $\mathrm{Cl}^{-}$ have been described previously in molluscs. The permeability of these receptor channels varies, with channels showing either a high degree of selectivity for $\mathrm{Cl}^{-}$(Chiarandini et al., 1967), or a wider range, including permeability to $\mathrm{PRO}^{-}$and formate ions (Kehoe, 1972b). However, none appear to have such a high permeability to $\mathrm{PRO}^{-}$as the present case. Further study of the permeability of this receptor ion channel will have to await characterization under voltage clamp.

Thus, the action of CARB on the EPSP can be simply explained as resulting from a reduction in the postsynaptic giant axon $R_{\mathrm{IN}}$; unlike the GLU agonists, it does not block the receptors activated by the endogenous transmitter. It is unlikely that CARB affects transmitter release directly as no changes were detected in the resting or action potentials of the presynaptic nerve terminal. On the basis of the observations that CARB does not desensitize the postsynaptic membrane to the endogenous transmitter, and that the cholinergic receptor identified on the postsynaptic giant axon does not have characteristics compatible with an excitatory synapse, the evidence suggests strongly that $\mathrm{ACh}$ is not the excitatory transmitter at this synapse.

We do not know the role of the cholinergic receptors on the postsynaptic giant axon. Despite the depolarization of the cholinergic agonists, the above evidence, together with the localization of $\mathrm{ACh}, \mathrm{CAT}$, and cholinesterases in the region of the giant synapse, would suggest that there is a neural input onto the giant axon that is inhibitory. Such an influence could be involved in the suppression of third-order axon excitability, or perhaps in a feedback inhibition circuit.

What, then, is the transmitter at the giant synapse? Despite the contrary evidence on the $E_{\mathrm{R}}$ referred to above, GLU or another amino acid must still be regarded as the best transmitter candidate. Only GLU (Miledi, $1967,1969)$ and its agonists, or aspartate and its agonists (Stanley, 1983), have been demonstrated both to depolarize the postsynaptic giant axon and also to interfere with the EPSP. Clearly, further studies will be necessary to resolve this question.

\section{References}

Abbott, N. J., M. Bundgaard, and H. F. Cserr (1981) Fine structural evidence for a glial blood-brain barrier to protein in the cuttlefish, Sepia officinalis. J. Physiol. (Lond.) 316: 52-53P.

Abbott, N. J., M. Bundgaard, and H. F. Cserr (1982) Experimental study on the blood-brain barrier in the cuttlefish, Sepia officinalis. J. Physiol. (Lond.) 326: 43-44P.

Blankenship, J. E., H. Wachtel, and E. R. Kandel (1971) Ionic mechanisms of excitatory, inhibitory, and dual synaptic actions mediated by an identified interneuron in abdominal ganglion of Aplysia. J. Neurophysiol. 34: 56-68.

Bryant, S. H. (1958) Transmission in squid giant synapses: The importance of oxygen and the effect of drugs. J. Gen. Physiol. 41: 473-484.

Bryant, S. H., and M. Brzin (1966) Cholinesterase activity of isolated giant synapses. J. Cell Physiol. 68: 107-108.

Brzin, M., V. M. Tennyson, and W-. D. Dettbarn (1975) Cytochemical localization of cholinesterase activity at the giant synapse of the squid. Histochemistry 43: 305-311.

Bullock, T. H. (1948) Properties of a single synapse in the stellate ganglion of the squid. J. Neurophysiol. 42: 609-616.

Bullock, T. H., and S. Hagiwara (1957) Intracellular recording from the giant synapse of the squid. J. Gen. Physiol. 40: 565$577,1957$.

Charlton, M. P., S. J. Smith, and R. S. Zucker (1982) Role of presynaptic calcium ions and channels in synaptic facilitation and depression at the squid giant synapse. J. Physiol. (Lond.) 323: 173-193.

Chiarandini, D. J., E. Stefani, and H. M. Gerschenfeld (1967) Ionic mechanisms of cholinergic excitation in molluscan neurons. Science 156: 1597-1599.

Froesch, D., and R. Martin (1972) Heterogeneity of synaptic vesicles in the squid giant fiber system. Brain Res. 43: 573579 .

Ginsborg, B. L. (1967) Ion movements in junctional transmission. Pharmacol. Rev. 19: 289-316. 
Heumann, R., J. Villegas, and D. W. Herzfeld (1981) Acetylcholine synthesis in the Schwann cell and axon in the giant nerve fiber of the squid. J. Neurochem. 36: 765-768.

Katz, B., and R. Miledi (1967) A study of synaptic transmission in the absence of nerve impulses. J. Physiol. (Lond.) 192: $407-436$.

Kehoe, J. (1972a) Three acetylcholine receptors in Aplysia neurones. J. Physiol. (Lond.) 225: 115-146.

Kehoe, J. (1972b) Ionic mechanisms of a two-component cholinergic inhibition in aplysia. J. Physiol. (Lond.) 225: 85-114.

Kelly, J. S., and P. W. Gage (1969) L-Glutamate blockade of transmission at the giant synapse of the squid stellate ganglion. J. Neurobiol. 2: 209-219.

Keynes, R. D. (1963) Chloride in the squid giant axon. J. Physiol. (Lond.) 169: 690-705.

Kusano, K., D. R. Livengood, and R. Werman (1967) Correlation of transmitter release with membrane properties of the presynaptic fiber of the squid giant synapse. J. Gen. Physiol. 50: 2579-2601.

Lester, M. A. (1970) Transmitter release by presynaptic impulses in the squid stellate ganglion. Nature 227: 493-496.

Llinas, R., R. W. Joyner, and C. Nicholson (1974) Equilibrium potential for the postsynaptic response in the squid giant synapse. J. Gen. Physiol. 64: 519-535.

Llinas, R., and C. Nicholson (1975) Calcium role in depolarization-secretion coupling: An aequorin study in squid giant synapse. Proc. Natl. Acad. Sci. U. S. A. 72: 187-190.

Llinas, R. L., I. Z. Steinberg, and K. Walton (1981) Relationship between presynaptic calcium current and postsynaptic potential in squid giant synapse. Biophys. J. 33: 323-352.

Miledi, R. (1967) Spontaneous synaptic potentials and quantal release of transmitter in the stellate ganglion of the squid. $\mathrm{J}$. Physiol. (Lond.) 192: 379-406.

Miledi, R. (1969) Transmitter action in the giant synapse of the squid. Nature 223: 1234-1235.

Miledi, R. (1972) Synaptic potentials in nerve cells of the stellate ganglion of the squid. J. Physiol. (Lond.) 225: 501-
514.

Miledi, R. (1973) Transmitter release induced by injection of calcium ions into the nerve terminal. Proc. R. Soc. Lond. Biol. 183: 421-425.

Nachmanson, D., and B. Meyerhof (1941) Relation between electrical change during nerve activity and concentration of cholinesterase. J. Neurophysiol. 4: 348-359.

Pumplin, D. W., and T. S. Reese (1978) Membrane ultrastructure of the giant synapse of the squid Loligo pealei. Neuroscience 3: 685-696.

Rosenberg, P., W.-D. Dettbarn, and M. Brzin (1966) Acetylcholine and choline acetylase in squid giant axon, ganglia and retina. Nature 210: 858-859.

Russell, J. M. (1976) ATP-dependent chloride influx into internally dialyzed squid giant axons. J. Membr. Biol. 28: 335349.

Stanley, E. F. (1983) Depolarizing and desensitizing actions of glutaminergic and cholinergic agonists at the squid giant synapse. Biol. Bull. 165: 533.

Stanley, E. F., and W. J. Adelman, Jr. (1982) Perfusion of the squid stellate ganglion through its blood supply: Implications for morphological and physiological studies of the squid giant synapse. Biol. Bull. 163: 403.

Takeuchi, A., and N. Takeuchi (1962) Electrical changes in pre- and postsynaptic axons of the giant synapse of Loligo. J. Gen. Physiol. 45: 1181-1193.

Webb, G. D., W. D. Dettbarn, and M. Brzin (1966) Biochemical and pharmocological aspects of the synapses of the squid stellate ganglia. Biochem. Pharmacol. 15: 1813-1814.

Williams, L. W. (1909) The Anatomy of the Common Squid, Brill, Leiden, Holland.

Yeh, J. Z., and T. Narahashi (1974) Noncholinergic mechanisms of action of cholinergic drugs on squid axon membranes. J. Pharmacol. Exp. Ther. 189: 697-707.

Young, J. Z. (1939) Fused neurons and synaptic contacts in the giant nerve fibres of cephalopods. Philos. Trans. R. Soc. Lond. Biol. 229: 465-503. 